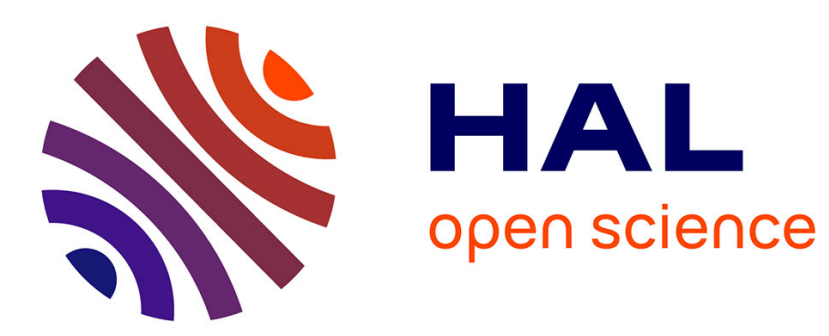

\title{
Kinematic properties of passive scalar gradient predicted by a stochastic Lagrangian model \\ M Gonzalez
}

\section{To cite this version:}

M Gonzalez. Kinematic properties of passive scalar gradient predicted by a stochastic Lagrangian model. Physics of Fluids, 2009, 21, pp.055104. 10.1063/1.3140004 . hal-01415347

\section{HAL Id: hal-01415347 \\ https://hal.science/hal-01415347}

Submitted on 13 Dec 2016

HAL is a multi-disciplinary open access archive for the deposit and dissemination of scientific research documents, whether they are published or not. The documents may come from teaching and research institutions in France or abroad, or from public or private research centers.
L'archive ouverte pluridisciplinaire HAL, est destinée au dépôt et à la diffusion de documents scientifiques de niveau recherche, publiés ou non, émanant des établissements d'enseignement et de recherche français ou étrangers, des laboratoires publics ou privés. 


\title{
Kinematic properties of passive scalar gradient predicted by a stochastic Lagrangian model
}

\author{
M. Gonzalez ${ }^{\text {a) }}$ \\ CNRS, UMR 6614/CORIA, Site universitaire du Madrillet, 76801 Saint-Etienne du Rouvray, France
}

(Received 29 July 2008; accepted 15 April 2009; published online 20 May 2009)

\begin{abstract}
A modeled equation for the gradient of a passive scalar is derived consistently with the approach of Chevillard and Meneveau for the velocity gradient tensor [Phys. Rev. Lett. 97, 174501 (2006)] and its predictions are analyzed in three-dimensional, isotropic turbulence. General features of scalar gradient kinematics, namely, production of scalar gradient norm and alignment with respect to strain principal axes and vorticity are rather well described. A strain persistence parameter defined for tight alignment of vorticity with a strain eigenvector is used to bring further insight into geometric properties of the scalar gradient. In particular, the model results lend support to the existence of special alignments of the scalar gradient which are determined by local strain persistence and are different from the directions of strain principal axes as already shown in two-dimensional turbulence. (C) 2009 American Institute of Physics. [DOI: 10.1063/1.3140004]
\end{abstract}

\section{INTRODUCTION}

The need of a precise understanding of stirring and mixing in turbulent flows has given rise to a number of studies focusing on the small-scale structure of scalar fields. ${ }^{1-4}$ The overall mixing mechanism including production of small scales of the scalar field and, eventually, smoothing down by molecular diffusion can be tackled through the behavior of the scalar gradient. In this view, mixing of scalar patches within the fluid medium finds expression in the rise of the scalar gradient caused by stretching (stirring) followed by diffusive damping (molecular mixing). In fact, the very process of micromixing makes stirring and molecular diffusion act together over a significant range of scales.

Micromixing efficiency is represented by the mean dissipation rate of the energy of scalar fluctuations, $\left\langle\epsilon_{\theta}\right\rangle$, which is proportional to the variance of the fluctuating scalar gradient. Analysis of its equation ${ }^{5}$ shows that $\left\langle\epsilon_{\theta}\right\rangle$ productionand thus sustained micromixing - is only ensured by the action of strain which results in the positive mean stretching term, $\left\langle-G_{\alpha} S_{\alpha \beta} G_{\beta}\right\rangle$, where the $G_{i}$ 's and $S_{i j}$ 's are the components of the scalar gradient, $\boldsymbol{G}$, and strain tensor, $S$, respectively. Enhancement of scalar gradient promoting stirring thus rests on both strain intensity and orientation of scalar gradient with respect to strain principal axes which is expressed by

$$
\begin{aligned}
-G_{\alpha} & S_{\alpha \beta} G_{\beta} \\
& =-|\boldsymbol{G}|^{2}\left[\lambda_{1} \cos ^{2}\left(\boldsymbol{G}, \boldsymbol{e}_{1}\right)+\lambda_{2} \cos ^{2}\left(\boldsymbol{G}, \boldsymbol{e}_{2}\right)+\lambda_{3} \cos ^{2}\left(\boldsymbol{G}, \boldsymbol{e}_{3}\right)\right],
\end{aligned}
$$

where the $\lambda_{i}$ 's are the strain eigenvalues with $\lambda_{3} \leq \lambda_{2} \leq \lambda_{1}$, $\lambda_{1} \geq 0$, and $\lambda_{3} \leq 0$. As a consequence of incompressibility, $\lambda_{1}+\lambda_{2}+\lambda_{3}=0$. Vectors $\boldsymbol{e}_{i}$ 's are the strain eigenvectors. Equation (1) clearly shows that scalar gradient production is a matter of intensity of strain components through the $\lambda_{i}$ 's val-

\footnotetext{
${ }^{a)}$ Electronic mail: michel.gonzalez@ coria.fr. Telephone: 33 (0)2 32953705. FAX: $33(0) 232910485$.
}

ues and of scalar gradient orientation in the strain basis through the director cosines. Clearly, production is promoted by alignment with the compressional direction, $\boldsymbol{e}_{3}$.

Even for the gradient of a passive scalar, the mechanisms underlying production are not as simple as Eq. (1) may suggest. Orientation of scalar gradient in the strain basis ${ }^{4}$ - see also Ref. 6 for the case of the material line-results from the complex combination of strain, vorticity, strain basis rotation, and molecular diffusion; through the equation for the strain tensor, strain basis rotation itself depends on strain, vorticity, pressure, and viscous effects ${ }^{4,6,7}$ and so do the strain eigenvalues. Moreover interaction between strain and vorticity makes this picture most intricate.

Accounting for such detailed mechanisms in small-scale mixing predictions needs devising models which, instead of being developed from the moment approach, ${ }^{5,8}$ are directly based on the behavior of the scalar gradient. In the present study this way was examined by extending the recent stochastic model of Chevillard and Meneveau ${ }^{9}$ for the velocity gradient tensor to the gradient of a passive scalar. The work is a first step that is chiefly focused on geometric properties underlying the production mechanisms of the scalar gradient. The main features of the model and the numerical method are given in Sec. II. Section III is devoted to results and analysis. Properties of scalar gradient predicted by the model such as alignments with strain eigenvectors and vorticity as well as production of gradient norm are reported. Then the model is used to get further insight into statistical alignment of the scalar gradient. Conclusion is drawn in Sec. IV.

\section{STOCHASTIC MODEL FOR THE GRADIENT OF A PASSIVE SCALAR}

\section{A. Derivation of a modeled equation for the scalar gradient}

The model for the passive scalar gradient is directly derived from the stochastic model for the velocity gradient developed by Chevillard and Meneveau. ${ }^{9}$ The latter model is 
based on an Eulerian-Lagrangian change in variables and the recent fluid deformation closure leading to the following modeled equation for the velocity gradient, $\boldsymbol{A}$ :

$d \boldsymbol{A}=\left(-\boldsymbol{A}^{2}+\frac{\operatorname{Tr}\left(\boldsymbol{A}^{2}\right)}{\operatorname{Tr}\left(\boldsymbol{C}_{\tau_{\eta}}^{-1}\right)} \boldsymbol{C}_{\tau_{\eta}}^{-1}-\frac{\operatorname{Tr}\left(\boldsymbol{C}_{\tau_{\eta}}^{-1}\right)}{3 T} \boldsymbol{A}\right) d t+\left(\frac{2}{T}\right)^{1 / 2} d \boldsymbol{W}$.

Stretching is exactly accounted for by the first term on righthand side, while the second and third ones model the pressure Hessian and viscous effects, respectively. They both include tensor $\boldsymbol{C}_{\tau_{\eta}}$ which is a model for the Cauchy-Green tensor; the latter is assumed to evolve in a frozen velocity gradient field during a time considered to be of the order of the Lagrangian decorrelation time scale of $\boldsymbol{A}$ that is, of the order of the Kolmogorov time scale, $\tau_{\eta}$. The modeled Cauchy-Green tensor, then, is expressed in terms of matrix exponentials as $\boldsymbol{C}_{\tau_{\eta}}=\exp \left(\tau_{\eta} \boldsymbol{A}\right) \exp \left(\tau_{\eta} \boldsymbol{A}^{T}\right)$. The time scale used for modeling the viscous term is the integral time scale, $T$; in the following, time is normalized by $T$. Tensorial noise $d \boldsymbol{W}$ represents the effects which are discarded by the previous models such as large-scale effects or the action of neighboring eddies. It is assumed to be Gaussian and white in time and is written as $d \boldsymbol{W}=d t^{1 / 2} \boldsymbol{\zeta}$, where $\boldsymbol{\zeta}$ is a tensorial, Gaussian delta-correlated noise with $\left\langle\zeta_{i j}\right\rangle=0$ and $\left\langle\zeta_{i j} \zeta_{k l}\right\rangle=2 \delta_{i k} \delta_{j l}$ $-1 / 2 \delta_{i j} \delta_{k l}-1 / 2 \delta_{i l} \delta_{j k}$, which ensures that $d \boldsymbol{W}$ is consistent with a traceless, isotropic tensor. This model has been shown to retrieve a number of geometric properties and anomalous scalings of turbulent flows; its predictions have been extensively compared to direct numerical simulation data. ${ }^{10}$

The exact equation for the gradient, $\boldsymbol{G}$, of a scalar is

$$
\frac{d \boldsymbol{G}}{d t}=-\boldsymbol{A}^{T} \boldsymbol{G}+D \nabla^{2} \boldsymbol{G},
$$

where $D$ is the molecular diffusivity of the scalar in the fluid. Provided that the velocity gradient is known-for instance, through Eq. (2) - the stretching term (first on right-hand side) does not need to be modeled. A model, however, has to be devised for the molecular diffusion term. This is done consistently with the approach that Chevillard and Meneveau' developed to model the velocity gradient equation.

The method thus essentially consists in modeling the Lagrangian Laplacian of $\boldsymbol{G}$ by a linear damping term. First, the Eulerian and Lagrangian Hessian's of $\boldsymbol{G}$ are related as

$$
\frac{\partial^{2} \boldsymbol{G}}{\partial x_{i} \partial x_{j}}=\frac{\partial X_{\alpha}}{\partial x_{i}} \frac{\partial X_{\beta}}{\partial x_{j}} \frac{\partial^{2} \boldsymbol{G}}{\partial X_{\alpha} \partial X_{\beta}},
$$

neglecting the spatial variations of $\partial \boldsymbol{X} / \partial \boldsymbol{x}$. In the mapping between Eulerian and Lagrangian coordinates $\boldsymbol{x}(\boldsymbol{X}, t)$ is the position at time $t$ of a fluid particle that was initially located at $\boldsymbol{x}\left(\boldsymbol{X}, t_{0}\right)=\boldsymbol{X}$ at time $t_{0}$. The Eulerian molecular diffusion term is therefore written as

$$
D \frac{\partial^{2} \boldsymbol{G}}{\partial x_{\gamma} \partial x_{\gamma}}=\frac{\partial X_{\alpha}}{\partial x_{\gamma}} \frac{\partial X_{\beta}}{\partial x_{\gamma}}\left(D \frac{\partial^{2} \boldsymbol{G}}{\partial X_{\alpha} \partial X_{\beta}}\right) .
$$

Second, the Lagrangian Hessian of $\boldsymbol{G}$ is assumed to be isotropic and is modeled by a linear damping term

$$
D \frac{\partial^{2} \boldsymbol{G}}{\partial X_{i} \partial X_{j}}=-\frac{1}{T_{\theta}} \frac{\delta_{i j}}{3} \boldsymbol{G},
$$

in which $T_{\theta}$ is the relaxation time scale. The modeled Eulerian molecular diffusion term, then, is

$$
D \nabla^{2} \boldsymbol{G}=-\frac{1}{3 T_{\theta}} \frac{\partial X_{\alpha}}{\partial x_{\beta}} \frac{\partial X_{\alpha}}{\partial x_{\beta}} \boldsymbol{G} .
$$

From the definition of the Cauchy-Green tensor, ${ }^{9} \boldsymbol{C}$, $\partial X_{\alpha} / \partial x_{\beta} \cdot \partial X_{\alpha} / \partial x_{\beta}=\operatorname{Tr}\left(\boldsymbol{C}^{-1}\right)$ in which, as previously explained, tensor $\boldsymbol{C}$ is modeled by $\boldsymbol{C}_{\tau_{\eta}}$. Finally, the model for the Eulerian molecular diffusion term is derived as

$$
D \nabla^{2} \boldsymbol{G}=-\frac{\operatorname{Tr}\left(\boldsymbol{C}_{\tau_{\eta}}^{-1}\right)}{3 T_{\theta}} \boldsymbol{G},
$$

and Eq. (3) for the gradient, $\boldsymbol{G}$, of a passive scalar is modeled as

$$
d \boldsymbol{G}=-\left(\boldsymbol{A}^{T} \boldsymbol{G}+\frac{\operatorname{Tr}\left(\boldsymbol{C}_{\tau_{\eta}}^{-1}\right)}{3 T_{\theta}} \boldsymbol{G}\right) d t+\left(\frac{2}{T_{\theta}}\right)^{1 / 2} d \boldsymbol{W}_{\boldsymbol{G}} .
$$

In Eq. (4) stretching by the velocity gradient (first term on right-hand side) is exact. Molecular diffusion is modeled by the second term and the third term represents random forcing. The latter is written as $d \boldsymbol{W}_{\boldsymbol{G}}=d t^{1 / 2} \boldsymbol{\xi}$, where $\boldsymbol{\xi}$ is a vectorial, Gaussian noise such that $\left\langle\xi_{i}\right\rangle=0$ and $\left\langle\xi_{i} \xi_{j}\right\rangle=\delta_{i j}$; this forcing is independent from the forcing of the velocity gradient. The relaxation time scale used to model molecular diffusion of the scalar gradient is based on the assumptions that (i) it coincides with the transfer time scale, $T_{\theta}$, of the scalar variance and (ii) the scalar-to-velocity time scale ratio, $T_{\theta} / T$, is constant. The latter assumption has been widely discussed, ${ }^{5,11-14}$ but is reasonable in a number of flows; in particular, the numerical simulations by Eswaran and Pope ${ }^{12}$ showed that in stationary, isotropic turbulence $T_{\theta} / T$ tends toward a constant value. Note that in the isotropic case the value of $T_{\theta} / T$ is connected to the scalar-to-velocity microscale ratio through $T_{\theta} / T=5 / 3 \mathrm{Sc} \lambda_{\theta}^{2} / \lambda^{2}$, where $\mathrm{Sc}$ is the Schmidt number, $\lambda$ is the Taylor microscale, and $\lambda_{\theta}$ is the scalar microscale. Finally, assuming $T_{\theta}$ being proportional to the integral time scale is consistent with Schmidt number of order unity or smaller; accounting for large Schmidt number would need making $T_{\theta}$ Reynolds number dependent through the Kolmogorov time scale. ${ }^{15}$

\section{B. Numerical solution}

The model is used by solving Eqs. (2) and (4) which generate stationary, velocity gradient and scalar gradient time signals. With the integral time scale, $T$, set to unity running the model needs to prescribe two parameters, namely, $\tau_{\eta}$ and $T_{\theta}$ or in other words, the Reynolds number and the scalar-tovelocity time scale ratio. Time scale $\tau_{\eta}$ is given the value 0.1 which corresponds to a Taylor Reynolds number close to $150 .{ }^{10}$ The scalar-to-velocity time scale ratio is $T_{\theta}=0.4$, a standard value for this kind of flow and near-unity Schmidt number. ${ }^{12}$ It has been found that increasing $T_{\theta}$ makes the convergence of scalar gradient statistics difficult. This problem is most likely to be ascribed to the linear model for the 


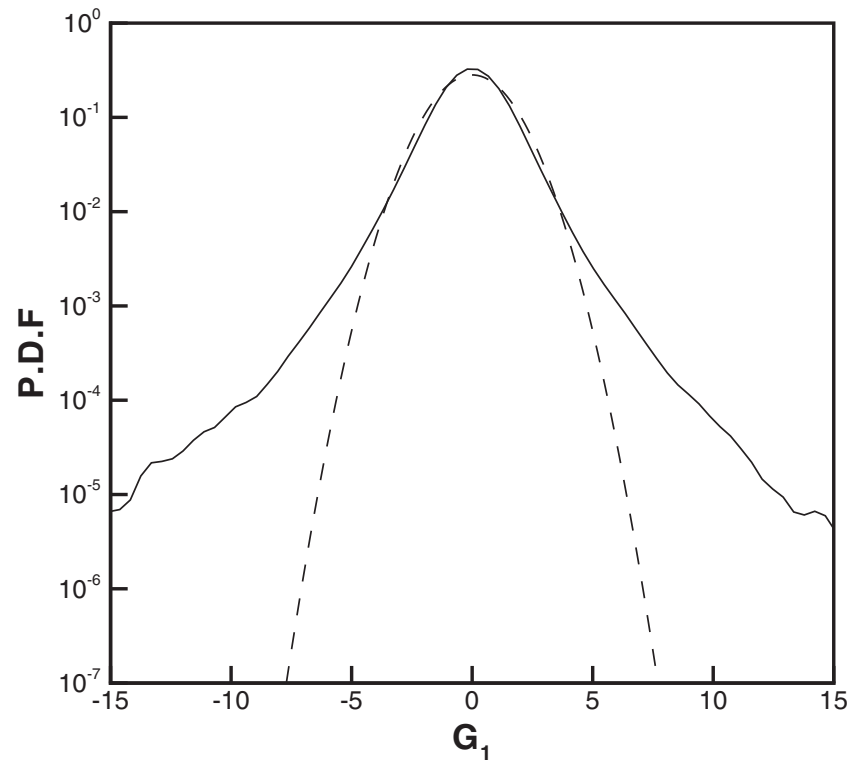

FIG. 1. p.d.f of component $G_{1}$ of scalar gradient; dashed line is the Gaussian law with the same variance.

molecular diffusion of the scalar gradient which uses the global, mean time scale $T_{\theta}$ as a relaxation time scale and may thus not correctly represent the way in which the actual dissipation balances production by instantaneous stretching. A more refined model should probably use a more "local" relaxation time scale, but the present model gives reliable results for moderate values of $T_{\theta}$.

The numerical procedure consists in using a secondorder predictor-corrector scheme ${ }^{16}$ to solve Eqs. (2) and (4). The calculation is run for $2 \times 10^{5} \mathrm{~T}$ with time step $10^{-2}$ and statistics of variables under study are derived from their respective time signals.

\section{STATISTICS AND KINEMATICS OF SCALAR GRADIENT}

\section{A. Comparison with previous studies}

Statistics of both velocity gradient and scalar gradient confirm isotropy. In particular, the results show that the even moments of the different components of $\boldsymbol{G}$ up to fourth order are equal, while odd moments and covariances are negligible. In addition, $\left\langle A_{11} G_{1}^{2}\right\rangle /\left\langle G_{\alpha} S_{\alpha \beta} G_{\beta}\right\rangle \simeq 0.127$ which is close to the isotropic value, $2 / 15,{ }^{17}$ within $5 \%$.

The probability density functions (p.d.f's) of scalar gradient components (Fig. 1) display the super-Gaussian tails reported by a number of authors. ${ }^{1,2,18,19}$ The normalized flatness of scalar gradient components, $\left\langle G_{i}^{4}\right\rangle /\left\langle G_{i}^{2}\right\rangle^{2}$, is about 7.30 , a somewhat underestimated value; according to the experimental data gathered by Warhaft, ${ }^{2}$ it should rather be around 10 for the present Reynolds number.

General results on production and geometric statistics agree well with previous studies. Figures 2 and 3 clearly show the positive skewness of the production of scalar gradient norm. ${ }^{4,17,20}$ In particular, the normalized production rate (Fig. 3) is very similar to that derived by Brethouwer et $a l{ }^{4}$ from numerical simulation. Moreover, the usual statistics

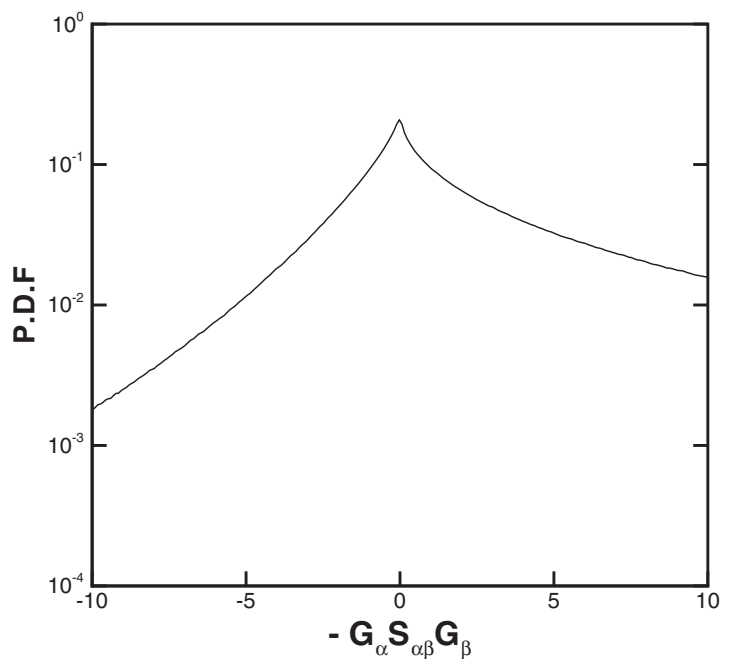

FIG. 2. p.d.f of the production term of scalar gradient norm.

of scalar gradient alignment in strain basis are retrieved (Fig. 4). As expected, 3,17,21 the scalar gradient aligns better with the most compressional direction $(i=3)$ than with the other strain directions $(i=1$, extensional direction; $i=2$, "intermediate" direction). Because the scalar gradient is kinematically attracted by the compression axis, this result is actually not surprising. Note that the scalar gradient aligns slightly better with the extensional than with the intermediate strain eigenvector which seems to be a special feature of Navier-Stokes turbulence-when compared to synthetic Gaussian flow. $^{22}$

More detailed results on alignment statistics are given in Figs. 5-7. As what Gulitski et al. ${ }^{17} \mathrm{did}$, these statistics are conditioned on $\left\langle\omega^{2}\right\rangle,\left\langle S^{2}\right\rangle$, and $\left\langle G^{2}\right\rangle$-with $\omega^{2}=\omega_{\alpha} \omega_{\alpha}$, $S^{2}=S_{\alpha \beta} S_{\beta \alpha}$, and $G^{2}=G_{\alpha} G_{\alpha}$. Conditional alignments of $\boldsymbol{G}$ with the strain eigenvectors (Figs. 5 and 6) show the right trends. In particular, the results clearly display more marked alignments for larger scalar gradient magnitude. However, in comparison to the data of Gulitski et al., ${ }^{17}$ this effect is magnified by the model. In the plots of the latter authors the maxima in the p.d.f of $\cos \left(\boldsymbol{G}, \boldsymbol{e}_{1}\right)$ (Fig. 6) are also much

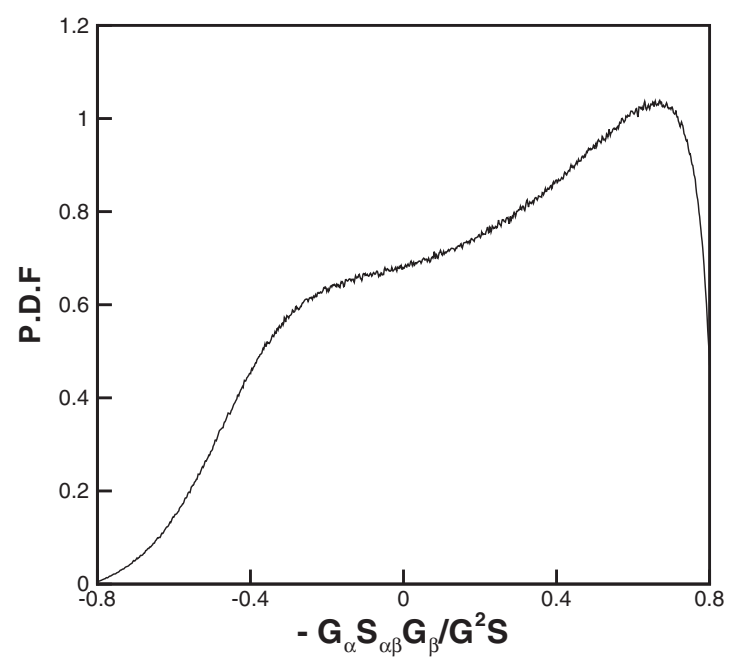

FIG. 3. p.d.f of the normalized production term of scalar gradient norm. 


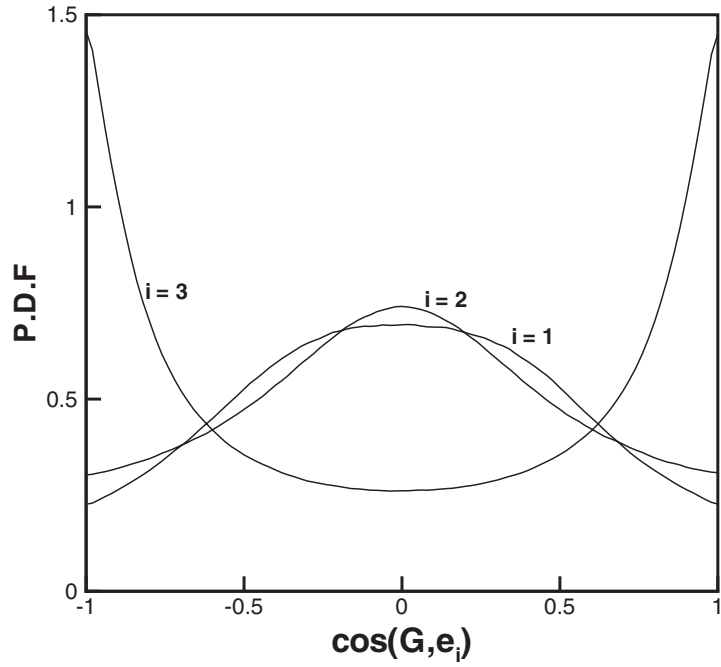

FIG. 4. p.d.f's of scalar gradient director cosines in strain basis; $i=1$, extensional direction; $i=2$, intermediate direction; $i=3$, compressional direction.

smaller. Finally, as shown in Fig. 7, the trend of the scalar gradient to align normally to vorticity is retrieved by the model and conditioned p.d.f's of $\cos (\boldsymbol{G}, \boldsymbol{\omega})$ agree rather well with those derived by Gulitski et al. ${ }^{17}$

\section{B. Further analysis of scalar gradient alignment}

When vorticity aligns close to a strain eigenvector a strain persistence parameter can be defined and used to determine rigorously whether the flow is locally strain or rotation dominated. ${ }^{23}$ If vorticity is aligned with $e_{1}$ the strain persistence parameter is defined as

$$
r_{1}=-\frac{\omega_{1}^{\prime}}{\lambda_{2}-\lambda_{3}}
$$

with $\omega_{1}^{\prime}=\hat{\omega}_{1}-\Omega_{1}$, where $\Omega_{1}=-2 \hat{\Pi}_{23} /\left(\lambda_{2}-\lambda_{3}\right)$ is a component of the rotation rate of strain principal axes; $\Pi$ is the pressure Hessian tensor defined by $\Pi_{i j}=(1 / \rho) \partial^{2} p / \partial x_{i} \partial x_{j}$, where $\rho$ and $p$ represent density and pressure, respectively. Hatted quantities indicate components in the strain basis. Note that accounting for effective rotation-i.e., the sum of vorticity and rotation rate of strain basis-in strain persistence was proposed by Dresselhaus and Tabor ${ }^{6}$ and that a strain persistence criterion including effective rotation has been defined by Tabor and Klapper ${ }^{24}$ and Lapeyre et al. ${ }^{25}$ in two-dimensional flows.

Analysis shows that the scalar gradient tends toward the plane normal to $\boldsymbol{e}_{1} \cdot{ }^{23}$ If $r_{1}^{2} \leq 1$ strain dominates and there is a stable, equilibrium orientation, $\boldsymbol{e}_{\mathrm{eq}}$, in the plane $\left(\boldsymbol{e}_{2}, \boldsymbol{e}_{3}\right)$ for the scalar gradient which depends only on $r_{1}$ through $\zeta_{\text {eq }}$ $=-\arccos r_{1} ; \zeta_{\mathrm{eq}}=2 \theta_{\mathrm{eq}}-\pi / 2$, where $\theta_{\mathrm{eq}}$ is the angle between $\boldsymbol{e}_{3}$ and $\boldsymbol{e}_{\text {eq }}$. The smaller $\left|r_{1}\right|$, the closer this orientation to the compressional direction, $\boldsymbol{e}_{3}$; both directions coincide for $r_{1}=0$. If $r_{1}^{2}>1$ effective rotation dominates and there is no stable orientation for the scalar gradient in the plane $\left(\boldsymbol{e}_{2}, \boldsymbol{e}_{3}\right)$, but a most probable one, $\boldsymbol{e}_{\text {prob }}$, depending on $r_{1}$ through $\zeta_{\text {prob }}=\left[1-\operatorname{sign}\left(r_{1}\right)\right] \pi / 2$, where $\zeta_{\text {prob }}=2 \theta_{\text {prob }}-\pi / 2$ and $\theta_{\text {prob }}$ is

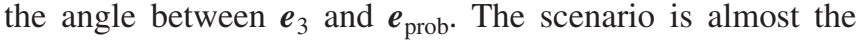
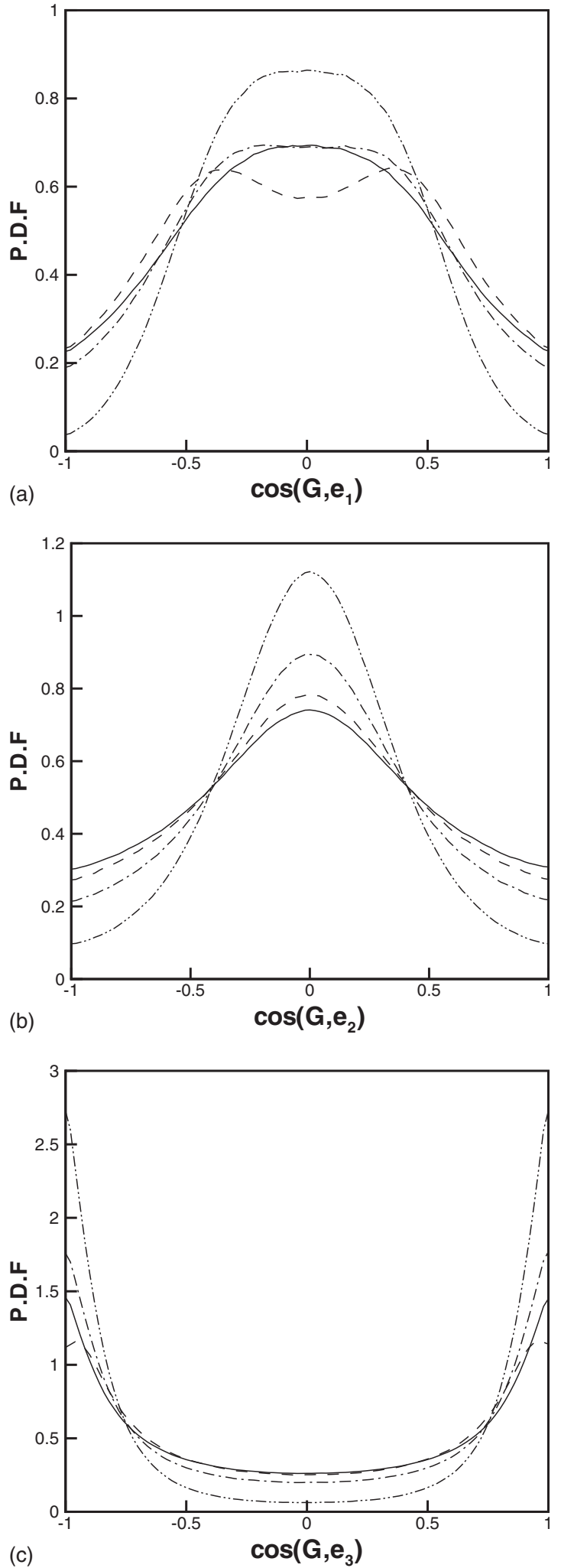

FIG. 5. p.d.f's of $\cos \left(\boldsymbol{G}, \boldsymbol{e}_{i}\right)$ conditioned on $\left.\omega^{2}\right\rangle\left\langle\omega^{2}\right\rangle$ (dashed), $\left.S^{2}\right\rangle\left\langle S^{2}\right\rangle$ (dot-dashed), and $\left.G^{2}\right\rangle\left\langle G^{2}\right\rangle$ (dot-dot-dashed); solid line: whole field; $i=1$, extensional direction; $i=2$, intermediate direction; $i=3$, compressional direction.

same for vorticity aligned with $\boldsymbol{e}_{2}$. The difference is that in the case of dominating strain the scalar gradient does not tend unconditionally toward the plane normal to $\boldsymbol{e}_{2}$, but only if $\left(1-r_{2}^{2}\right)^{1 / 2}+3 \lambda_{2} /\left(\lambda_{1}-\lambda_{3}\right)>0$, where $r_{2}$ is the strain persis- 

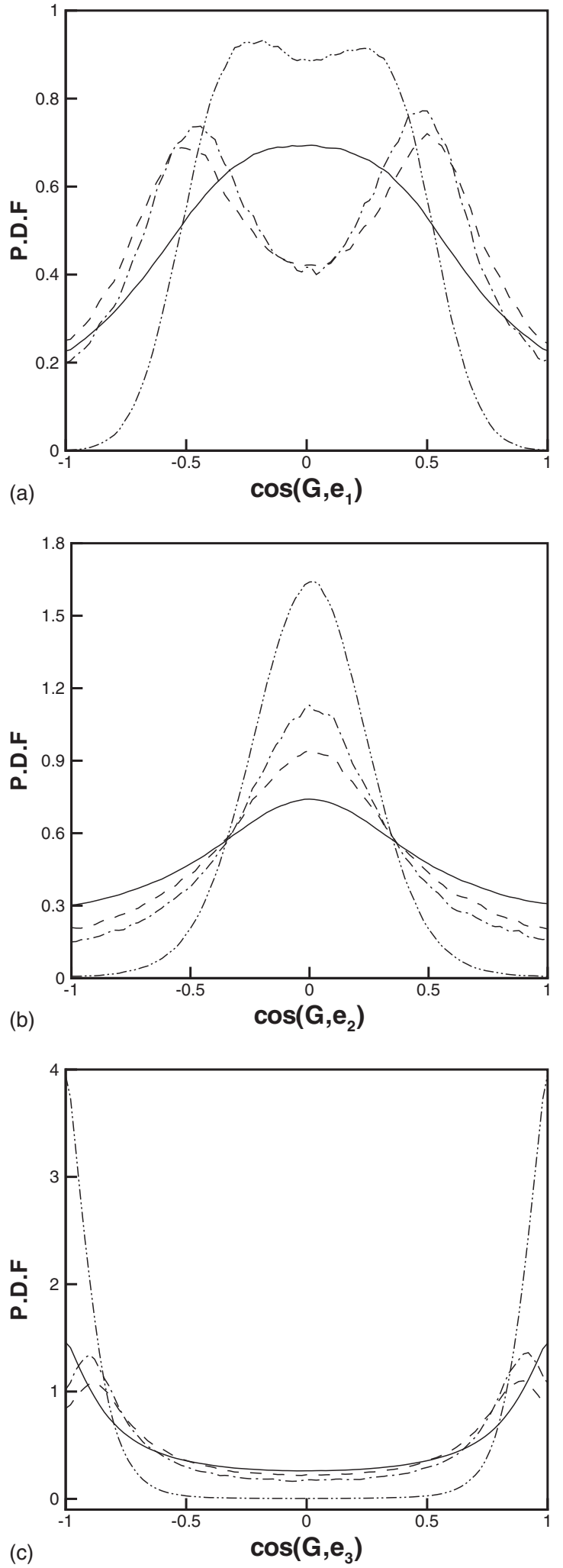

FIG. 6. p.d.f's of $\cos \left(\boldsymbol{G}, \boldsymbol{e}_{i}\right)$ conditioned on $\omega^{2}>3\left\langle\omega^{2}\right\rangle$ (dashed), $S^{2}>3\left\langle S^{2}\right\rangle$ (dot-dashed), and $G^{2}>3\left\langle G^{2}\right\rangle$ (dot-dot-dashed); solid line: whole field; $i=1$, extensional direction; $i=2$, intermediate direction; $i=3$, compressional direction.

tence parameter, $r_{2}=\omega_{2}^{\prime} /\left(\lambda_{1}-\lambda_{3}\right)$, with $\omega_{2}^{\prime}=\hat{\omega}_{2}-\Omega_{2}$ and $\Omega_{2}$ $=2 \hat{\Pi}_{13} /\left(\lambda_{1}-\lambda_{3}\right){ }^{23}$ The analysis can be done for vorticity aligned with $\boldsymbol{e}_{3}$ as well.

Since the above definition of strain persistence param-
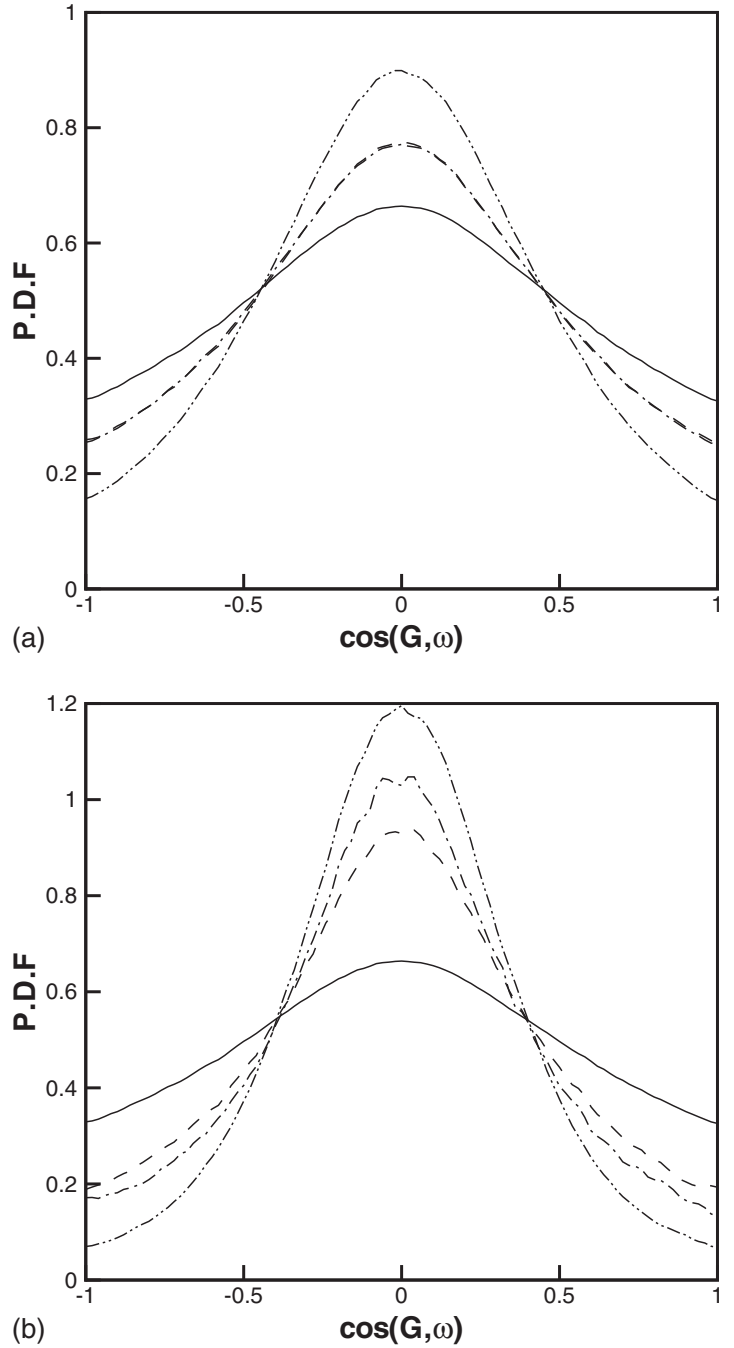

FIG. 7. p.d.f of $\cos (\boldsymbol{G}, \boldsymbol{\omega})$; (a) conditioned on $\left.\omega^{2}\right\rangle\left\langle\omega^{2}\right\rangle$ (dashed), $\left.S^{2}\right\rangle\left\langle S^{2}\right\rangle$ (dot-dashed), and $\left.G^{2}\right\rangle\left\langle G^{2}\right\rangle$ (dot-dot-dashed); solid line: whole field; (b) conditioned on $\omega^{2}>3\left\langle\omega^{2}\right\rangle$ (dashed), $S^{2}>3\left\langle S^{2}\right\rangle$ (dot-dashed), and $G^{2}>3\left\langle G^{2}\right\rangle$ (dot-dot-dashed); solid line: whole field.

eter, $r_{i}$, is valid in the three-dimensional case provided that vorticity tightly aligns with strain eigenvector $\boldsymbol{e}_{i},{ }^{23}$ the condition for computing $r_{i}$ has been defined as $\left|\cos \left(\boldsymbol{\omega}, \boldsymbol{e}_{i}\right)\right|$ $\geq 0.99$ which is denoted by $\boldsymbol{\omega} / / \boldsymbol{e}_{i}$. In so far as vorticity does statistically align with strain eigenvectors, ${ }^{21,26}$ such an analysis is relevant to turbulent flow structure. It is also relevant to the vortex model approach ${ }^{27,28}$ in which vorticity is assumed to be fully aligned with a straining direction.

Figure 8 displays the p.d.f's of strain persistence parameters $r_{i}(i=1,2,3)$ computed in samples where $\boldsymbol{\omega} / / \boldsymbol{e}_{i}$. The p.d.f's of $r_{1}$ and $r_{2}$ strongly peak around -1 and 1 which suggests that strain and effective rotation mostly balance each other in regions where vorticity aligns close to $\boldsymbol{e}_{1}$ or $\boldsymbol{e}_{2}$. For $\boldsymbol{\omega} / / \boldsymbol{e}_{1}$, however, a larger part of the sample corresponds to dominating effective rotation. More precisely, strain- and rotation-dominated regions amount to $39 \%$ and $61 \%$, respectively, when $\boldsymbol{\omega} / / \boldsymbol{e}_{1}$ and $49 \%$ and $51 \%$ when $\boldsymbol{\omega} / / \boldsymbol{e}_{2}$. Interestingly, cases in which $\boldsymbol{\omega} / / \boldsymbol{e}_{3}$ are the most rotation dominated. Within the samples $\boldsymbol{\omega} / / \boldsymbol{e}_{i}$ cases $\boldsymbol{\omega} / / \boldsymbol{e}_{2}$ are the most prob- 


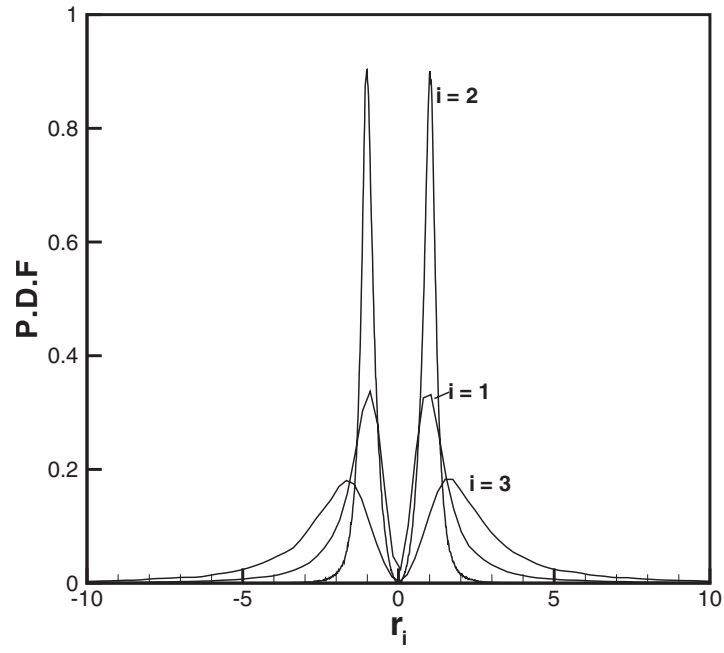

FIG. 8. p.d.f of strain persistence.

able, $78 \%$, while cases $\boldsymbol{\omega} / / \boldsymbol{e}_{1}$ and $\boldsymbol{\omega} / / \boldsymbol{e}_{3}$ amount to $14 \%$ and $8 \%$, respectively. The following analysis is restricted to cases $\boldsymbol{\omega} / / \boldsymbol{e}_{1}$ and $\boldsymbol{\omega} / / \boldsymbol{e}_{2}$.

Figures 9 and 10 show the strain- and rotationdominated samples for $\boldsymbol{\omega} / / \boldsymbol{e}_{1}$ and $\boldsymbol{\omega} / / \boldsymbol{e}_{2}$-determined by parameters $r_{1}$ and $r_{2}$, respectively-in the $\left(R^{*}, Q^{*}\right)$ plane where $Q^{*}=-1 / 2 A_{\alpha \beta} A_{\beta \alpha} /\left\langle S_{\alpha \beta} S_{\beta \alpha}\right\rangle$ and $R^{*}$ $=-1 / 3 A_{\alpha \beta} A_{\beta \gamma} A_{\gamma \alpha} /\left\langle S_{\alpha \beta} S_{\beta \alpha}\right\rangle^{3 / 2}$ are the second- and thirdorder invariants of the velocity gradient tensor, respectively. Because strain eigenvalue $\lambda_{1}$ is positive, while $\lambda_{2}$ may be either positive or negative, rotation-dominated data represent only stretched vorticity- $R^{*}<0$ and $Q^{*}>0$; "stable focus/stretching" $" 29$-in the $\boldsymbol{\omega} / / \boldsymbol{e}_{1}$ case and both stretched and compressed vorticity $-R^{*}>0$ and $Q^{*}>0$; "unstable focus/compressing" ${ }^{29}$-in the $\boldsymbol{\omega} / / \boldsymbol{e}_{2}$ case; in the latter case, stretched vorticity, however, is more probable than compressed vorticity as a result of the well-established feature that positive values of $\lambda_{2}$ are more probable than negative ones. ${ }^{21,26}$ It is worth noting that Chevillard et al. ${ }^{10}$ found that their model for the pressure Hessian does not retrieve the actual dynamics of $R^{*}$ and $Q^{*}$ in quadrants $R^{*}>0 ; Q^{*}>0$ and $R^{*}<0 ; Q^{*}<0$, which might indirectly affect the predicted behavior of the scalar gradient.

Analysis of scalar gradient alignment can now be pushed further using the strain persistence parameters, $r_{1}$ and $r_{2}$. In particular, alignment with directions $\boldsymbol{e}_{\text {eq }}$ and $\boldsymbol{e}_{\text {prob }}$ can be derived and compared to alignment with the compressional direction, $\boldsymbol{e}_{3}$. Orientation statistics are significantly different when vorticity aligns with either $\boldsymbol{e}_{1}$ or $\boldsymbol{e}_{2}$. As shown in Fig. 11 , for $\boldsymbol{\omega} / / \boldsymbol{e}_{2}$ and prevailing strain, the scalar gradient aligns better with the equilibrium orientation, $\boldsymbol{e}_{\mathrm{eq}}$, lying in the plane $\left(\boldsymbol{e}_{1}, \boldsymbol{e}_{3}\right)$ than with the compressional direction. When $\boldsymbol{\omega} / / \boldsymbol{e}_{1}$, by contrast, alignments with the compressional direction and the equilibrium direction in the plane $\left(\boldsymbol{e}_{2}, \boldsymbol{e}_{3}\right)$ are statistically equivalent. In fact, the compressional and equilibrium directions are closer to each other when $\boldsymbol{\omega} / / \boldsymbol{e}_{1}$ than when $\boldsymbol{\omega} / / \boldsymbol{e}_{2}$ (not shown); this may be explained by small values of $r_{1}$ (in the range $[-0.7,0.7]$ ) being slightly more probable than small values of $r_{2}$. Moreover, better alignment of scalar gradient with equilibrium orientation when $\boldsymbol{\omega} / / \boldsymbol{e}_{2}$ may result
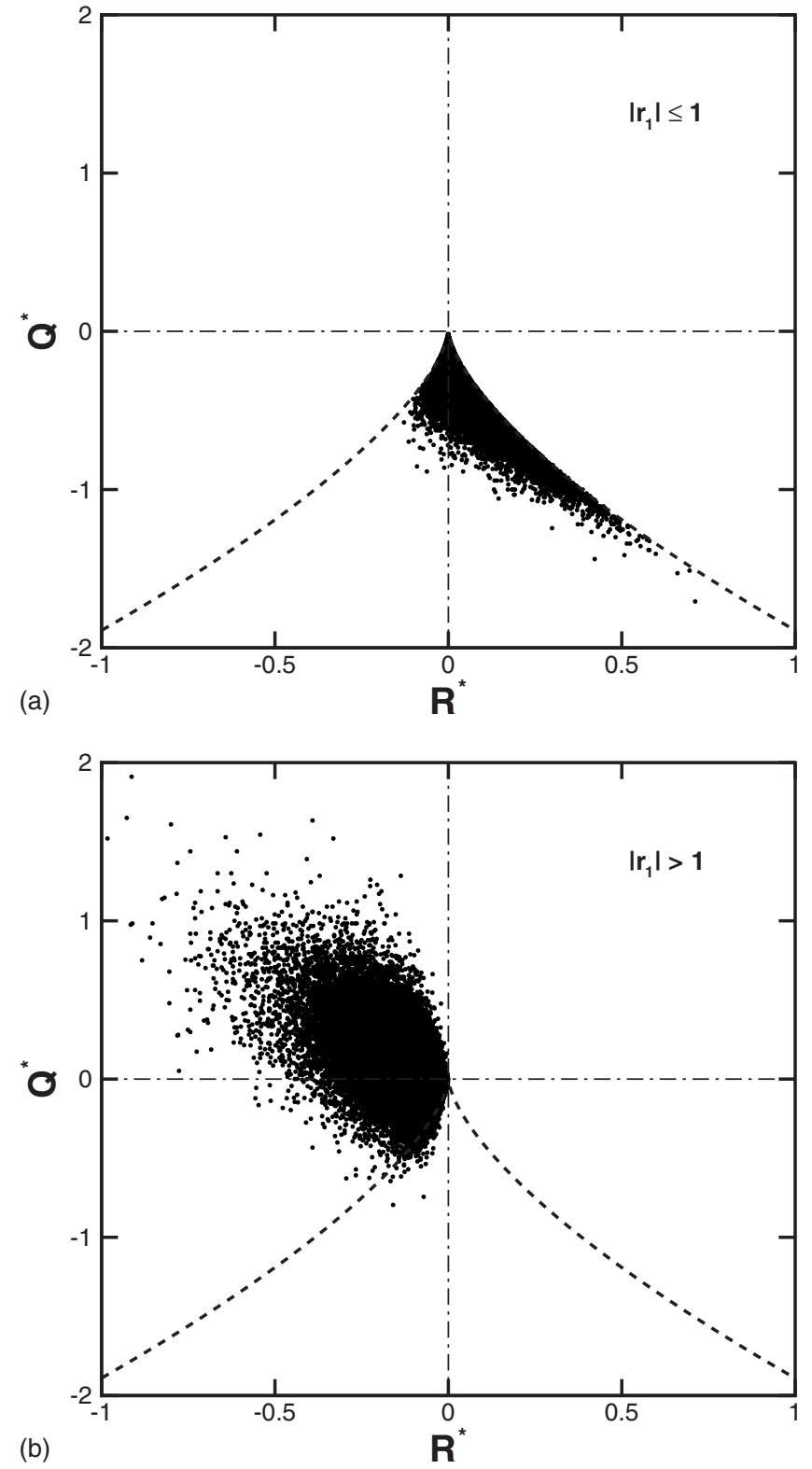

FIG. 9. Strain-dominated regions (a) and rotation-dominated regions (b) for $\boldsymbol{\omega} / / \boldsymbol{e}_{1}$-determined by strain persistence parameter, $r_{1}$, in the plane of second- and third-order invariants of velocity gradient; dashed line: $Q^{* 3}+27 R^{* 2} / 4=0$.

from values of strain time scale, $\left(\lambda_{1}-\lambda_{3}\right)^{-1}$, smaller than in the case $\boldsymbol{\omega} / / \boldsymbol{e}_{1}$ where this time scale is $\left(\lambda_{2}-\lambda_{3}\right)^{-1}$. Garcia et al. ${ }^{30,31}$ showed that in the two-dimensional case alignment of the scalar gradient is in fact determined by the gradient response to strain persistence fluctuations; more precisely, good alignment with the equilibrium direction needs that the variations of strain persistence occur on a time scale larger than the response time scale of the scalar gradient-which is roughly given by strain intensity. It is interesting to see that in this turbulent, three-dimensional case alignment with the equilibrium direction-computed for $\boldsymbol{\omega} / / \boldsymbol{e}_{1}$ and $\boldsymbol{\omega} / / \boldsymbol{e}_{2}$-is strong which is reminiscent of the results of Lapeyre et al. ${ }^{25}$ in turbulent, two-dimensional flows.

Almost similar results are found when rotation prevails (Fig. 12). Alignment with compressional direction, $\boldsymbol{e}_{3}$, is 

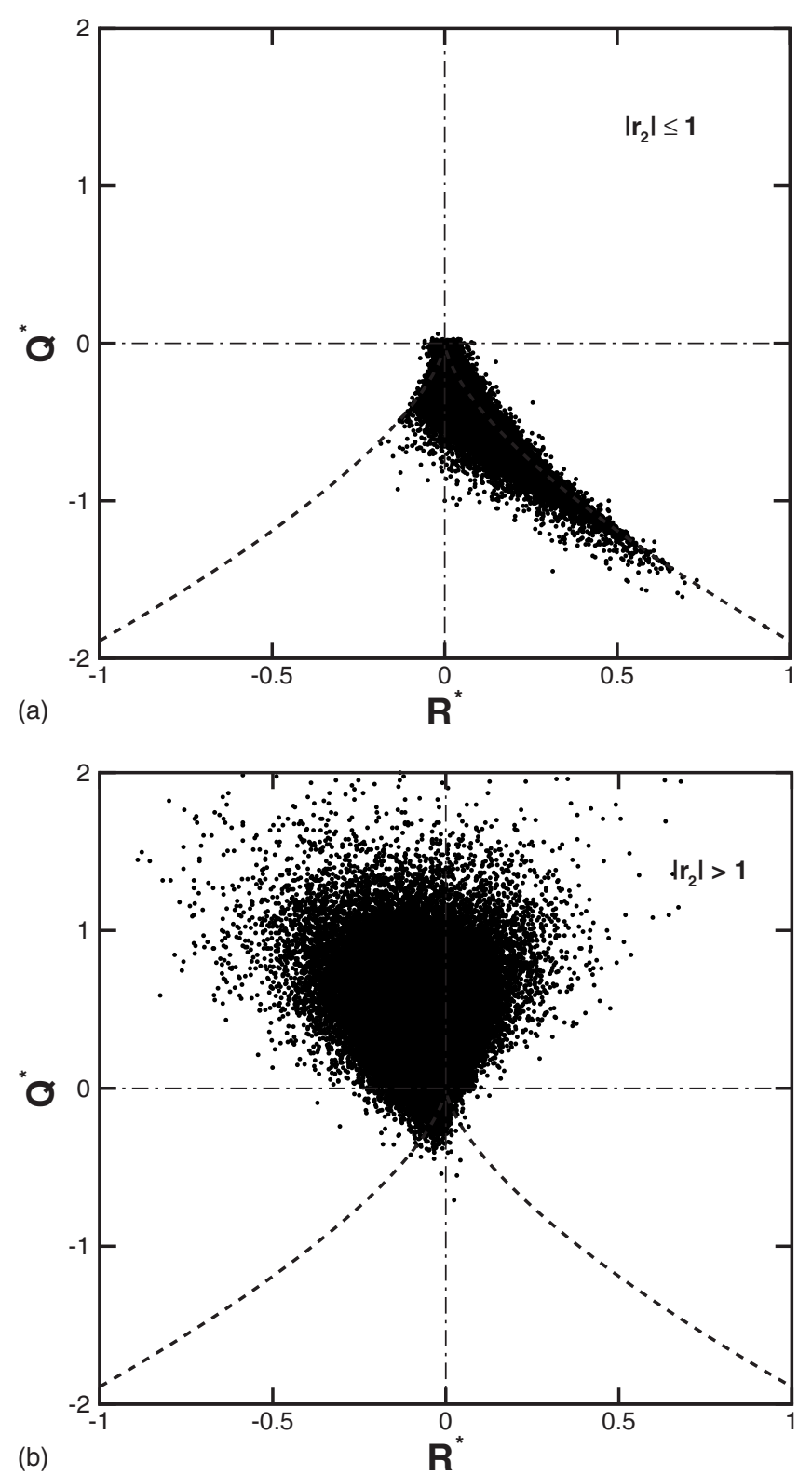

(b)

$\mathbf{R}^{*}$

FIG. 10. Strain-dominated regions (a) and rotation-dominated regions (b) for $\boldsymbol{\omega} / / \boldsymbol{e}_{2}$-determined by strain persistence parameter, $r_{2}$, in the plane of second- and third-order invariants of velocity gradient; dashed line: $Q^{* 3}+27 R^{* 2} / 4=0$.

slightly better than alignment with direction $\boldsymbol{e}_{\text {prob }}$ when $\boldsymbol{\omega} / / \boldsymbol{e}_{1}$; but when $\boldsymbol{\omega} / / \boldsymbol{e}_{2}$ alignment of the scalar gradient with direction $\boldsymbol{e}_{\text {prob }}$ determined by strain persistence is much better than alignment with the compressional direction. These results for both prevailing strain and prevailing rotation lend support to the existence of special alignments with directions depending on local strain persistence which are different from the strain principal axes as already shown in twodimensional turbulence. ${ }^{25}$

\section{CONCLUSION}

The stochastic model of Chevillard and Meneveau for the velocity gradient tensor has been extended to the gradient of a passive scalar and tested in three-dimensional, isotropic
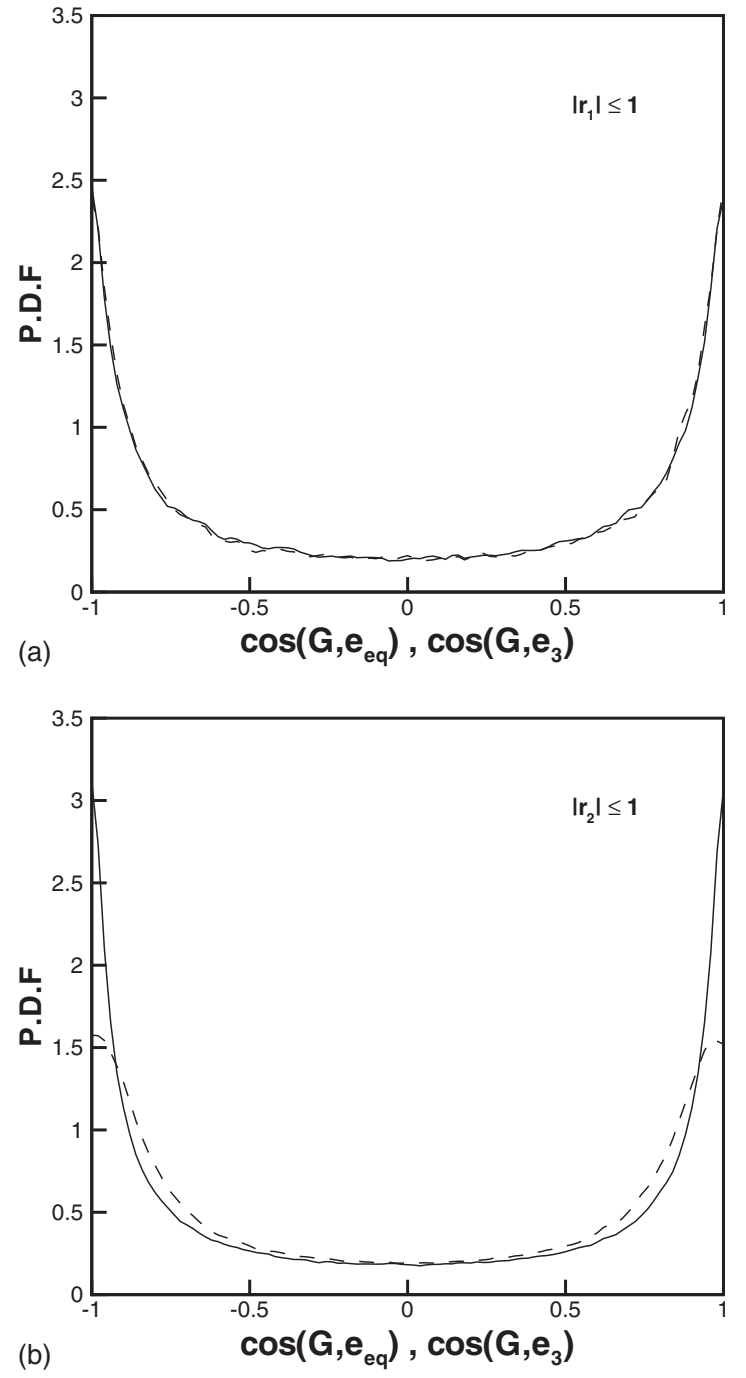

FIG. 11. p.d.f's of $\cos \left(\boldsymbol{G}, \boldsymbol{e}_{\mathrm{eq}}\right)$ (solid line) and $\cos \left(\boldsymbol{G}, \boldsymbol{e}_{3}\right)$ (dashed line) in strain-dominated regions; (a) $\boldsymbol{\omega} / / \boldsymbol{e}_{1} ;$ (b) $\boldsymbol{\omega} / / \boldsymbol{e}_{2}$.

turbulence. The concern of this work was more specifically in the analysis of kinematic properties of the scalar gradient.

General properties such as statistics of scalar gradient components, p.d.f's of production of scalar gradient norm as well as p.d.f's of alignment with strain eigenvectors and vorticity are retrieved by the model. The special features of statistical alignment with strain eigenvectors conditioned on the respective magnitudes of vorticity, strain or scalar gradient itself, however, seems to be magnified by the model.

The model has also been used to derive more detailed statistics on scalar gradient alignment. This has been achieved by using a strain persistence parameter which is defined in the case where vorticity aligns close to a strain eigenvector and which measures the respective influences of strain and effective rotation-vorticity plus rotation rate of strain basis. Model predictions suggest that the scalar gradient may take preferential alignments which are determined by local strain persistence and depart from the directions of strain principal axes. This behavior has already been stressed in two-dimensional turbulence, but has not been investigated in the three-dimensional case. These results would therefore 

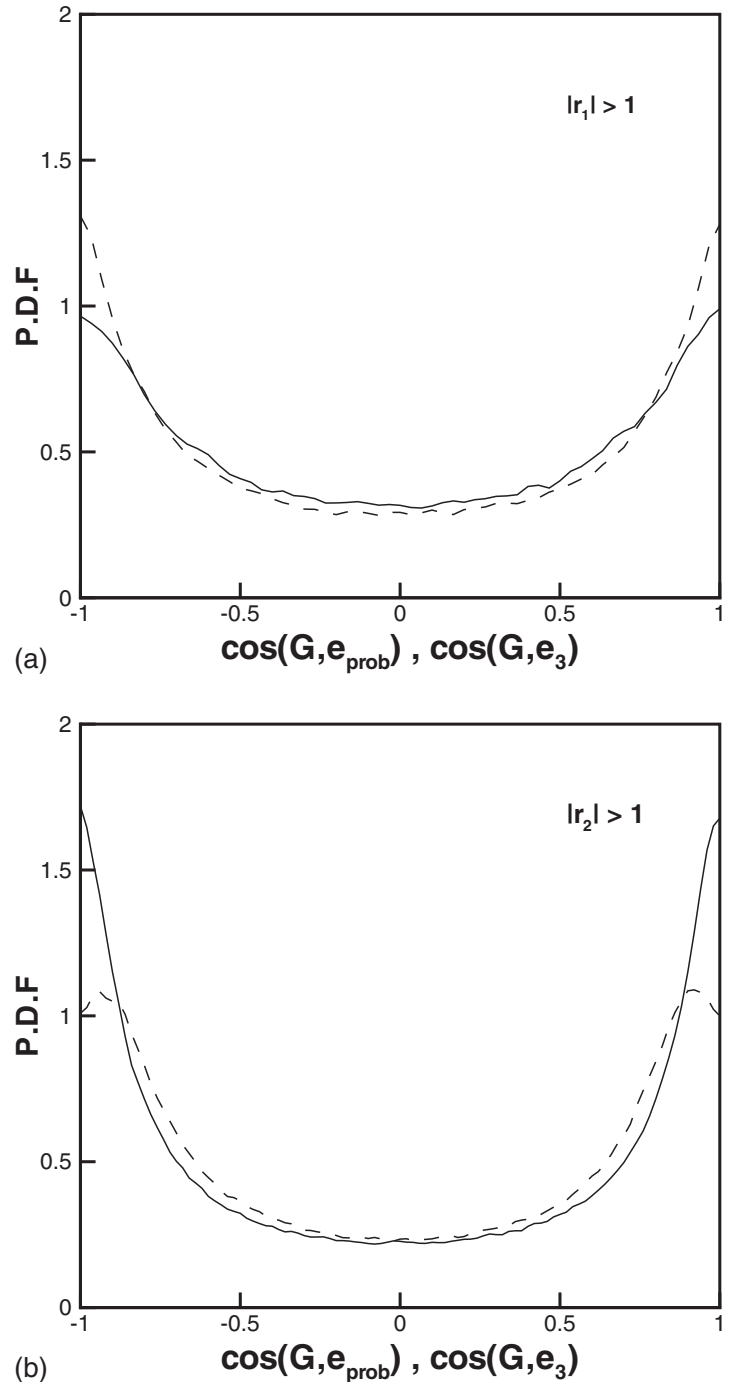

FIG. 12. p.d.f's of $\cos \left(\boldsymbol{G}, \boldsymbol{e}_{\text {prob }}\right)$ (solid line) and $\cos \left(\boldsymbol{G}, \boldsymbol{e}_{3}\right)$ (dashed line) in rotation-dominated regions; (a) $\boldsymbol{\omega} / / \boldsymbol{e}_{1}$; (b) $\boldsymbol{\omega} / / \boldsymbol{e}_{2}$

deserve to be appraised by different models, numerical simulation, or experimental data.

Further work should address different issues. First, model results for the scalar gradient obviously depend on the modeling of the velocity gradient tensor; it would therefore be worth checking to what extent the velocity gradient model indirectly influences the predicted scalar gradient properties. Second, the model has been derived, as a first step, for nearunity Schmidt number. Investigating the influence of Schmidt number is a key issue, but needs a different modeling of the molecular diffusion term and may be numerically more delicate. Finally, there is a number of experimental and numerical results in the case where forcing of the scalar field, instead of being isotropic, is achieved through a steady, mean scalar gradient. Accounting for anisotropic forcing of the scalar gradient would thus certainly give further insight into the behavior of the model.

\section{ACKNOWLEDGMENTS}

The author is grateful to Laurent Chevillard and Charles Meneveau for their advice and encouragement.
${ }^{1}$ A. Pumir, "A numerical study of the mixing of a passive scalar in three dimensions in the presence of a mean gradient," Phys. Fluids 6, 2118 (1994).

${ }^{2}$ Z. Warhaft, "Passive scalars in turbulent flows," Annu. Rev. Fluid Mech. 32, 203 (2000).

${ }^{3}$ P. Vedula, P. K. Yeung, and R. O. Fox, "Dynamics of scalar dissipation in isotropic turbulence: A numerical and modeling study," J. Fluid Mech. 433, 29 (2001).

${ }^{4}$ G. Brethouwer, J. C. R. Hunt, and F. T. M. Nieuwstadt, "Micro-structure and Lagrangian statistics of the scalar field with a mean gradient in isotropic turbulence," J. Fluid Mech. 474, 193 (2003).

${ }^{5}$ G. R. Newman, B. E. Launder, and J. L. Lumley, "Modelling the behaviour of homogeneous scalar turbulence," J. Fluid Mech. 111, 217 (1981).

${ }^{6} \mathrm{E}$. Dresselhaus and M. Tabor, "The kinematics of stretching and alignment of material elements in general flow fields," J. Fluid Mech. 236, 415 (1992).

${ }^{7}$ K. K. Nomura and G. K. Post, "The structure and dynamics of vorticity and rate of strain in incompressible homogeneous turbulence," J. Fluid Mech. 377, 65 (1998).

${ }^{8}$ D. B. Spalding, "Concentration fluctuations in a round turbulent free jet," Chem. Eng. Sci. 26, 95 (1971).

${ }^{9}$ L. Chevillard and C. Meneveau, "Lagrangian dynamics and statistical geometric structure of turbulence," Phys. Rev. Lett. 97, 174501 (2006).

${ }^{10}$ L. Chevillard, C. Meneveau, L. Biferale, and F. Toschi, "Modeling the pressure Hessian and viscous Laplacian in turbulence: Comparisons with direct numerical simulation and implications on velocity gradient dynamics," Phys. Fluids 20, 101504 (2008).

${ }^{11}$ C. Béguier, I. Dekeyser, and B. E. Launder, "Ratio of scalar and velocity dissipation time scales in shear flow turbulence," Phys. Fluids 21, 307 (1978).

${ }^{12}$ V. Eswaran and S. B. Pope, "Direct numerical simulation of the turbulent mixing of a passive scalar," Phys. Fluids 31, 506 (1988).

${ }^{13} \mathrm{M}$. Gonzalez and A. Fall, "The approach to self-preservation of scalar fluctuations decay in isotropic turbulence," Phys. Fluids 10, 654 (1998).

${ }^{14} \mathrm{M}$. Gonzalez, "Asymptotic evolution of a passive scalar field advected by an homogeneous turbulent shear flow," Int. J. Heat Mass Transfer 43, 387 (2000).

${ }^{15}$ S. Corrsin, "The isotropic turbulent mixer: Part II. Arbitrary Schmidt number," AIChE J. 10, 870 (1964).

${ }^{16}$ W. C. Welton and S. B. Pope, "PDF model calculations of compressible turbulent flows using smoothed particle hydrodynamics," J. Comput. Phys. 134, 150 (1997).

${ }^{17}$ G. Gulitski, M. Kholmyanski, W. Kinzelbach, B. Lüthi, A. Tsinober, and S. Yorish, "Velocity and temperature derivatives in high-Reynolds-number turbulent flows in the atmospheric surface layer. Part 3. Temperature and joint statistics of temperature and velocity derivatives," J. Fluid Mech. 589, 103 (2007).

${ }^{18}$ M. Holzer and E. D. Siggia, "Turbulent mixing of a passive scalar," Phys. Fluids 6, 1820 (1994).

${ }^{19} \mathrm{C}$. Tong and Z. Warhaft, "On passive scalar derivative statistics in grid turbulence," Phys. Fluids 6, 2165 (1994).

${ }^{20}$ G. R. Ruetsch and M. R. Maxey, "The evolution of small-scale structures in homogeneous isotropic turbulence," Phys. Fluids 4, 2747 (1992).

${ }^{21}$ W. T. Ashurst, A. R. Kerstein, R. M. Kerr, and C. H. Gibson, "Alignment of vorticity and scalar gradient with strain rate in simulated Navier-Stokes turbulence," Phys. Fluids 30, 2343 (1987).

${ }^{22}$ A. Tsinober and B. Galanti, "“"Exploratory numerical experiments on the differences between genuine and "passive" turbulence," Phys. Fluids 15, 3514 (2003).

${ }^{23}$ A. Garcia and M. Gonzalez, "Analysis of passive scalar gradient alignment in a simplified three-dimensional case," Phys. Fluids 18, 058101 (2006).

${ }^{24} \mathrm{M}$. Tabor and I. Klapper, "Stretching and alignment in chaotic and turbulent flows," Chaos, Solitons Fractals 4, 1031 (1994).

${ }^{25}$ G. Lapeyre, P. Klein, and B. L. Hua, "Does the tracer gradient vector align with the strain eigenvectors in 2D turbulence?," Phys. Fluids 11, 3729 (1999).

${ }^{26} \mathrm{~K}$. Ohkitani, "Numerical study of comparison of vorticity and passive vectors in turbulence and inviscid flows," Phys. Rev. E 65, 046304 (2002).

${ }^{27}$ J. M. Burgers, "Application of a model system to illustrate some points of the statistical theory of free turbulence," Proc. R. Acad. Sci. Amsterdam 43, 2 (1940). 
${ }^{28}$ T. S. Lundgren, "Strained spiral vortex model for turbulence fine structure," Phys. Fluids 25, 2193 (1982).

${ }^{29}$ M. S. Chong, A. E. Perry, and B. J. Cantwell, "A general classification of three-dimensional flow fields," Phys. Fluids 2, 765 (1990).

${ }^{30}$ A. Garcia, M. Gonzalez, and P. Paranthoën, "On the alignment dynamics of a passive scalar gradient in a two-dimensional flow," Phys. Fluids 17, 117102 (2005)

${ }^{31}$ A. Garcia, M. Gonzalez, and P. Paranthoën, "Nonstationary aspects of passive scalar gradient behaviour," Eur. J. Mech. B/Fluids 27, 433 (2008). 